\title{
FLOW CHARACTERISTICS OF INK-JET INKS USED FOR FUNCTIONAL PRINTING
}

Katarina Dimić-Mišić*

Aalto University, School of Chemical Technology, Finland

Alp Karakoc

Aalto University, School of Chemical Technology, Finland

Merve Ozkan

Aalto University, School of Chemical Technology, Finland

Syed Ghufran Hashmi

Aalto University, New Energy Technologies Group, Finland

Thad Maloney

Aalto University, School of Chemical Technology, Finland

Jouni Paltakari

Aalto University, School of Chemical Technology, Finland

The rheology-related effects of nozzle clogging in inkjet printing can seriously affect ink ejection rate and result in irregular droplet trajectory, resulting in poor printing results. In this study three different inkjet inks, used for functional printing with a Dimatix materials inkjet printer, are investigated rheologically. In connection with rheological observation a novel method of inkjet trajectory image analysis gives insight into the irregularity of directional deviation due to clogging of nozzles of the printer It is found that the type of solvent used in the ink formulation has an important influence on the rheological behaviour in the printing regime, which directly has an effect on ink-droplet trajectory and contact characteristic with the substrate.

Key words: Inkjet printing, Rheology of inkjet inks, Nozzle clogging, Ink droplet trajectory

\section{INTRODUCTION}

Inkjet printing (IP) is a versatile, non-contact, high speed and precise patterning technique which decreases the manufacturing costs of printable devices by minimising the waste and process time [03, 05]. Commercialisation of many research technologies on numerous substrates can only be realised by such an economic, ecologically-friendly and high throughput method [05]. Although especially drop-on-demand (DoD) IP is a very competitive mass production method it poses some limitations preventing it to be a standard part of the manufacturing lines especially due to the crucial ink requirements $[05,06]$. DoD process starts with an impulse is applied to the nozzle of the printer and a drop is formed within the limitations given by the nature of the ink and properties of the printer, followed by the ejection from the nozzle. The droplet, in some cases with undesirable 'satellite drops', travels between the printing orifice and the substrate [02]. Ideally, the satellite-free and round-shaped drop needs to follow a straight trajectory with a controlled velocity. Clogging of nozzles in inkjet printing is the most disruptive phenomenon in the sensitive operation of ink-jet printing, affecting print quality in several ways. Partial clogging can change the flow pattern and print out uniformity, and the internal property and quality of ejected ink can be seriously compromised, not least by a contamination derived from a clogging build-up breaking off and entering the ejected drop. While researchers have intensively used IP technology in the manufacture of many printed electronic devices, such as thin-film transistors (TFT) and organic light emitting diodes (OLED), there are still present problems related to the uneven prints, that are a result of nozzle choking, an effect of fast solvent evaporation and complex rheological behaviour of the IP inks [20].

Various types of IP inks have been developed with low viscosity solvents in order to increase performance of print out, especially when used for electronic devices applications. Reduction of 
fluidity of the inks through evaporation and gelation is important to prevent leakage and avoid undesirable contact with solvents, especially when sealing materials are present. In addition, too high an increase in viscosity of the IP ink used for printing electronics upon gelation leads to dilatant behaviour and can furthermore lead to undesirable clogging of the IP nozzles $[07,08]$.

In order to obtain high performance jetting of the functional IP inks used for Dimatix printer, optimised parameters define the desirable physical properties for the jetting of inks used for printed electronics: viscosity ( $\eta \sim 10-12 \mathrm{mPas})$, surface tension $\left(\sigma \sim 28-42 \times 10-3 \mathrm{Nm}^{-1}\right)$, boiling point (more than $100{ }^{\circ} \mathrm{C}$ ), density (more than $1 \mathrm{gcm}^{-3}$ ), $\mathrm{pH}$ value [04-09].

Using rheological evaluation of the three different IP-inks used for functional printing of electronic devices, discussed in this paper, in respect to their viscosity dependence over a broad shear rate range and their structure recovery, it is possible to correlate their flow parameters with the print out performance. Measuring ejected trajectory from a clean Dimatix nozzle, and a same nozzle after printing has been performed for a few days, we can observe that clogging of the nozzle due to the observed complex rheological behaviour results in a change in ejected trajectory [11-14].

\section{EXPERIMENTAL METHODS Characterisation of Inks}

Three types of organic solvents were used for production of functional printing IP inks, aimed for printing electronics: L-D (low-dilatant), M-D (medium dilatant) and $\mathrm{H}-\mathrm{D}$ (high dilatant), respectively. Solvents had different boiling temperatures of $285^{\circ} \mathrm{C}, 164^{\circ} \mathrm{C}$ and $82^{\circ} \mathrm{C}$, and surface tension values of 40,27 and $24 \mathrm{mNm}^{-1}$, respectively, measured using an optical tension meter (CAM 200 from KSV instruments) in pendant drop mode.

The surface tension of L-D, M-D and L-D exhibited surface tension values of $\sigma=40 \mathrm{mNm}^{-1}, 27$ $\mathrm{mNm}^{-1}, 24 \mathrm{mNm}^{-1}$, respectively. However, the viscosity was a more complex phenomena which was presented in detail below.

\section{Inkjet Printing Process}

A piezoelectric, laboratory scale DoD materials inkjet printer (Dimatix 2831-DMP) [14, 17, 20] was used to test the printability of the inks. The DMP employs a disposable cartridge which is made of chemically resistant polypropylene silicone and silicon dioxide. The cartridge is composed of 2 main components: jetting and storage units. Following a filtering step, and (for some cases) degassing, around $2 \mathrm{~cm}^{3}$ liquid is filled into the polypropylene bag in the printer storage unit with the help of a special syringe with a blunt tip needle and the two units were attached together. The regular cleaning cycles can be run before, during and after the printing process with contact made with a cleaning pad to maintain or improve the printing performance. The cleaning cycle consists of three actions: blotting, purging and jetting having adjustable order and time lengths [14].The substrate is placed on a special platen having equally spaced holes which provides vacuum to keep the substrate in the desired position. The platen temperature can be raised to $60-70{ }^{\circ} \mathrm{C}$. The ink is jetted upon an impulse applied to the jetting module of the cartridge, which is attached to the storage unit. In the jetting module 16 nozzles are embedded in a single row and each nozzle has a channel-type connection to the ink storage unit. The straightforward and flexible processability of DoD that operate at frequency, $1-20 \mathrm{kHz}$ [05-08]. The main limiting factors for such an operating condition are the fast evaporation of the solvent, resulting in choking of the nozzles, and increase in the viscosity $[14,20]$.

a)

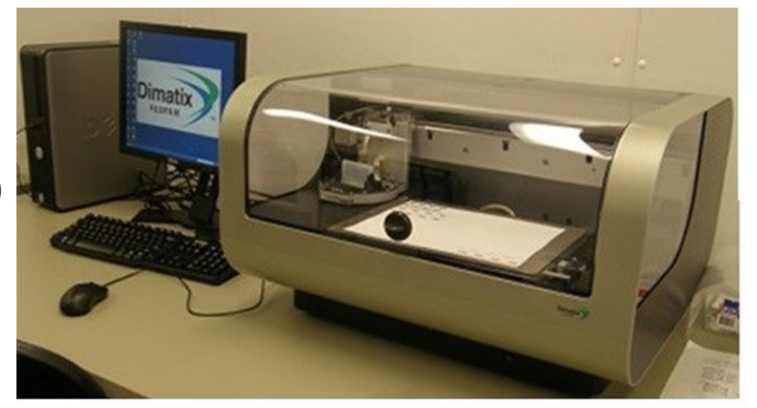

b)

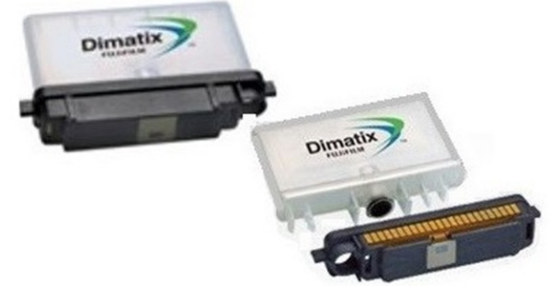

Figure 1: Fujifilm's Dimatix s printer DMP-2831 and his parts a), DoD inkjet printer, that allows the user to deposit fluid materials on an A4 sized substrate using piezo-based inkjet cartridge b) cartridge consists of storage unit and jetting (printing) head 


\section{Rheometry}

Rheological measurements were made to characterise the viscoelastic properties of the inks in different temperature and shear ranges.

\section{Linear and non-linear behaviour}

The viscoelastic rheological investigations were performed at $23^{\circ} \mathrm{C}$ by means of an Anton Paar 300 (Anton Paar Austria $\mathrm{GmbH}$ ) oscillatory constant stress/strain and variable shear rheometer. For rheological measurements of inks, an upper serrated plate-plate geometry PP-25 was selected, while the bottom plate was smooth, in order to reduce any potential wall-slip effect, gap was set to $0.5 \mathrm{~mm}$. In order to avoid any memory-effect from previous deformations all samples were pre-sheared under oscillation at an angular frequency $(\omega)$ of $10 \mathrm{rad} / \mathrm{s}^{-1}$ and strain deformation $(\mathrm{Y})$ of $0.01 \%$ for $10 \mathrm{~min}$, followed by a rest stationary state time of $15 \mathrm{~min}$. To prevent evaporation of the water medium, a layer of silicone oil was spread around the boundary of the sample in the geometry in contact with the air - a common procedure $[02,19]$. To perform the frequency sweep test, the linear viscoelastic range of the sample (LVE) was obtained from an amplitude sweep using constant angular frequency $(\omega$ $=1 \mathrm{~s}^{-1}$ ) with varying strain amplitude in the range $y=0.01-100 \%$. The influence of frequency on the variation of complex viscosity $\left(n^{*}\right)$, which is within the LVE range, was evaluated for angular frequency in the range $\omega=0.1-100 \mathrm{rad} / \mathrm{s}^{-1}$. Response of the dynamic viscosity ( $\eta$ ) to shear rate was measured over the shear rate range $y=$ $0.01-1000 \mathrm{~s}^{-1}$ applying a logarithmic data collection duration from $500-1 \mathrm{~s}$.

\section{Recovery and dilatancy measurements}

Structure recovery measurements were performed with the three interval test (3ITT test). To evaluate the evolution of $\eta$ after high shear stress, the 3ITT rotational test was performed consisting of a step-wise shear rate test with three defined intervals of applied shear. During the first and third intervals, the sample was sheared at a low shear rate of $0.1 \mathrm{~s}^{-1}$, following the second interval during which high shear, = $500 \mathrm{~s}^{-1}$. For evaluation of the tendency to dilatant behaviour, another 3ITT test was performed where in the second interval shear rate was linearly increased from 1 to $500 \mathrm{~s}^{-1}$, recording the dynamic viscosity $(\eta)$ response.

\section{Droplet Trajectory determination: regular and irregular ejection}

To investigate the influence of the rheological properties on the droplet trajectory, image processing was implemented to distinguish between the difference in droplet formation and trajectory Fig. 2a), at first when the nozzle was clean at the initial stage of printing, and Fig. 2b) when time has passed and the nozzle opening was choked leading to irregular ink ejection which changed droplet trajectory, as presented in Fig. 2c). Complex rheological behaviour may result also in lower performance as a function of the firing frequency, drop velocity and drop formation under deformation, creating irregular droplets or spherical drops of insufficient volume. The change in trajectory after drop formation was evaluated for the case where the nozzle was clean and after using the same nozzle for few days, due to the effect of the complex rheological behaviour and deposition of ink on the edge of nozzles, including unwanted evaporation.

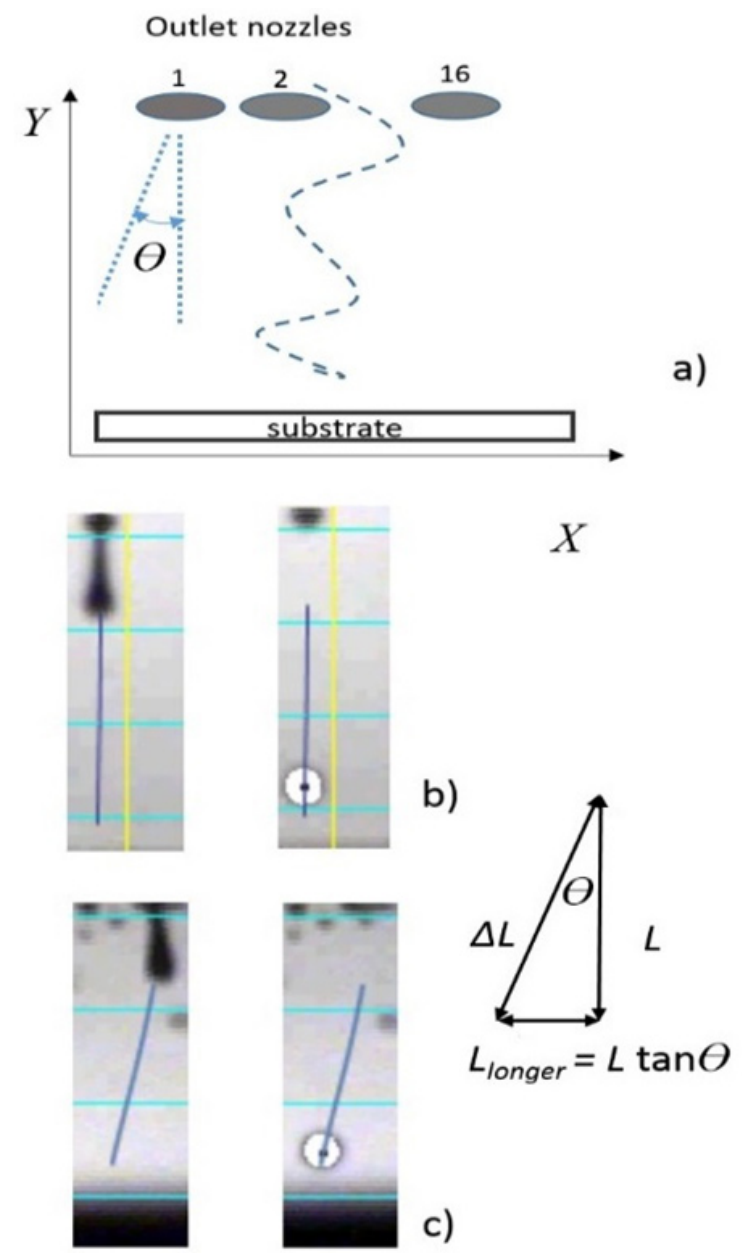

Figure 2: Droplet images taken from Dimatix nozzles: a) schematic presentation of the determination of trajectory deviation angle $\Delta \theta$ b) regular droplet trajectory when ink was ejected from clean nozzle, and c) irregular ejection seen 


\section{RESULTS}

\section{Rheological analysis}

For successful printing of IP inks, due to their fast evaporation at high temperatures, thermal and rheological stability under jetting are important $[12,13]$, especially when considering fast evaporation of solvent at the nozzle-air contact. For this reason, when engineering functional printing inks, used for printing electronics, with a lower colloidal volume fraction [16, 18], the tuning of rheological behaviour is very important in tailoring ink viscosity and drying kinetics. Fig. 3a)b) shows clearly that the complex and dynamic shear response behaviour as a function of shear rate is different to that of the complex response as a function of frequency, with the complex viscosity $\left(n^{*}\right)$ being much higher than the dynamic viscosity $(\eta)$, with both $\eta^{*}$ and $\eta$ revealing shear thinning properties at the lower energy input state but dilatant behaviour for successive alternate conditions of higher frequency $\left([17,20]\right.$. Both $\eta^{*}$ complex viscosity and $\eta$ dynamic viscosity, Fig. 4a)-b), for ink L-D reveal more stable flow curves that ink $M-D$ and $H-D$, which exhibited instability in both $\eta^{*}$ and $\eta$ attributed to viscoelastic fluctuations and dilatancy, respectively.
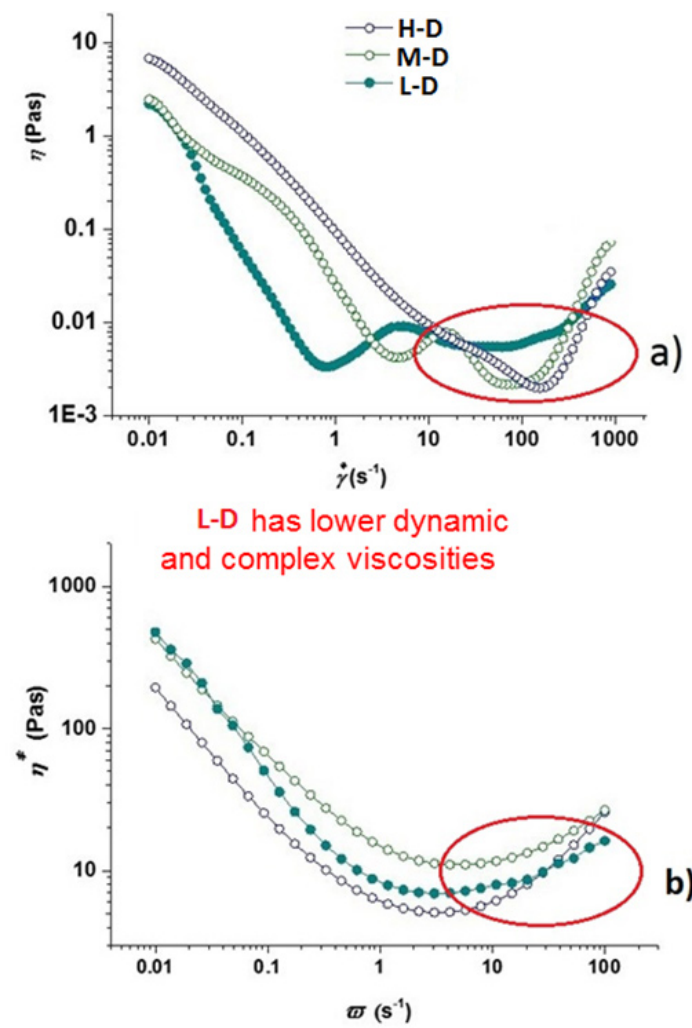

Figure 3: Rheological behaviour of inks: a) steady state flow curve for dynamic viscosity $D$,

b) viscoelastic response expressed through complex viscosity $\left(\eta^{*}\right)$ showing increase at higher frequencies, being lowest from $L-D$
The IP inks have shear thinning rheological behaviour, but since the solvent evaporates during the printing process their viscosity changes through the duration of use, depending on the process conditions [8-10]. As presented in Fig. $4 a)-b)$ the inks eventually display dilatant behaviour, and therefore it is important to define the degree of viscosity increase after the high shear period. However, as presented in Fig. 4a), viscosity increase resulting from the high shear rate interval is lowest for the L-D ink, which is important when considering that dilatant behaviour leads to clogging for nozzles and a related decrease in printing performance [20]. second 3ITT interval, viscosity decreases and then stays constant. When the shear force is decreased again, the viscosity increases again, as this is typical for thixotropic behaviour [2, 19]. Fig. 4b) shows the viscosity of inks when the shear rate is linearly increased between $0.1-500 \mathrm{~s}^{-1}$.
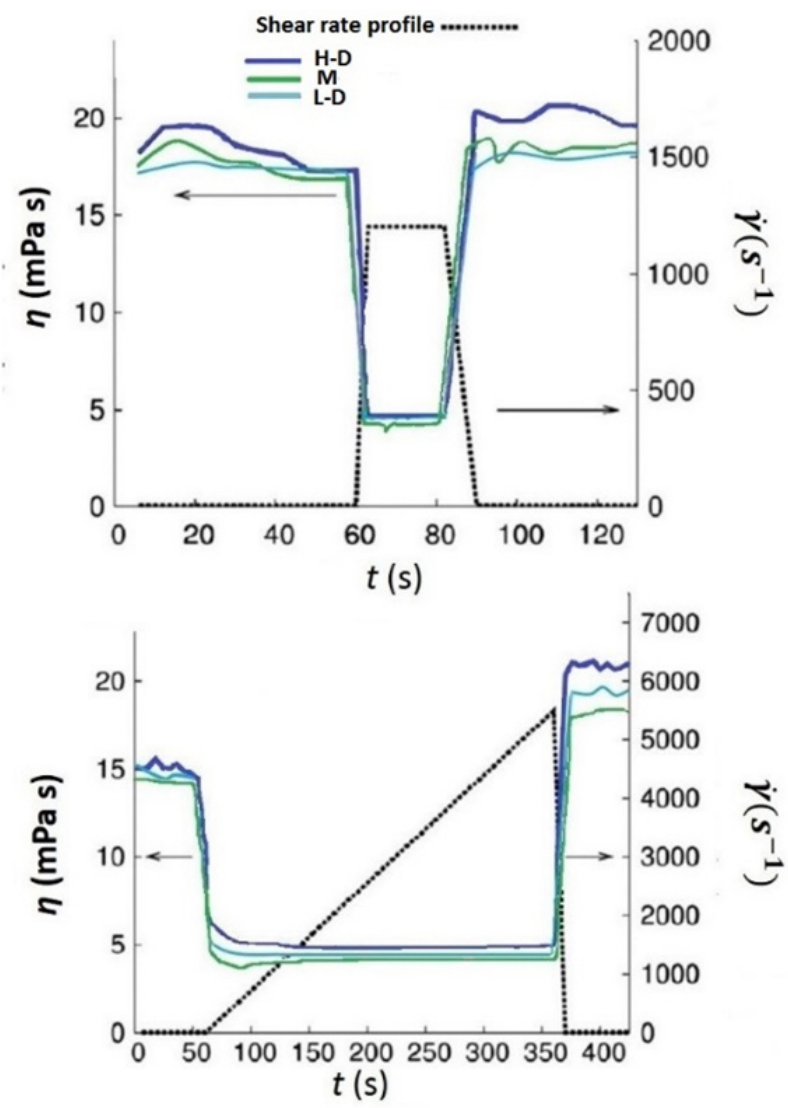

Figure 4: Response of $\eta$ in 3ITT DSSC inks: a) step wise constant shear rate, and b) a linearly increasing shear rate showing dilatant behaviour of inks

The more stable response of L-D can explain its good printability. explained as clogging of the nozzle cause by increased elasticity and dilatant viscosity accompanied with faster solvent evaporation, as presented in Table 1. 
Table 1: Rheological parameters of DSSC inks obtained from flow curves and structure recovery tests

\begin{tabular}{|l|c|c|c|}
\hline Characteristic Property & H-D & M-D & L-D \\
\hline$\eta$ @ $10^{\mathrm{s}-1}(\mathrm{mPa} . \mathrm{s})$ & 7.8 & 8.2 & 10 \\
\hline$\eta$ @ $100^{\mathrm{s}-1}(\mathrm{mPa} . \mathrm{s})$ & 2.4 & 3.4 & 9 \\
\hline$\eta$ @ $1000^{\mathrm{s}-1}(\mathrm{mPa} . \mathrm{s})$ & 58 & 62 & 24 \\
\hline$\eta$ @ after 3ITT, $(\dot{\gamma}=$ const $(\%))$ & 34 & 27 & 5 \\
\hline$\eta$ @ after 3ITT, $(\dot{\gamma}=$ linear increase (\%)) & 37 & 29 & 7 \\
\hline
\end{tabular}

The IP-ink printing performance was tested on Xerox paper for the clarity of information, as shown in Fig. 5a)-c). With paper as substrate it is clearly possible to see the difference between the ideal dot shape when it comes in contact with substrate "single spherical droplet's size before spreading" and dot printouts in respect to the solvent used for each ink $[13,16]$. Once the deposits of inks are formed they tend to clog inkjet print head nozzles due to further attractive build-up and reduced velocity of flow, which reduces print performance further and even requires frequent replacement of the ink supply channel.

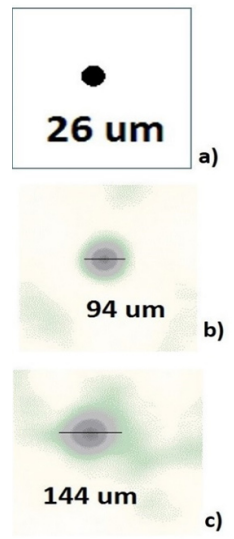

Figure 5: Inkjet printed inks on Xerox paper: a) "single spherical droplet's" b) spreading of ink solvent on paper due to uneven concertation within ink matrix, c) extreme spreading of ink due to uneven distribution of solven and ink particles on substrate

\section{Droplet trajectory}

In order to study the impact of rheology on the clogging of the nozzle and the effect it has on ink trajectory, in drop trajectory evaluation was carried using image processing from the images taken during the jetting process of M-D ink. In the current study, one cycle of the inkjet printing was taken into account, video frames of which images were extracted for one single nozzle and shown in Figure $6 a)$-c). Thereafter, extracted frames were digitised to form a binary image so as to obtain foreground and background pixel data as seen in Figure 6a). To determine the binary threshold, the Otsu method was used, which minimises the combined spread of foreground and background pixels [11].

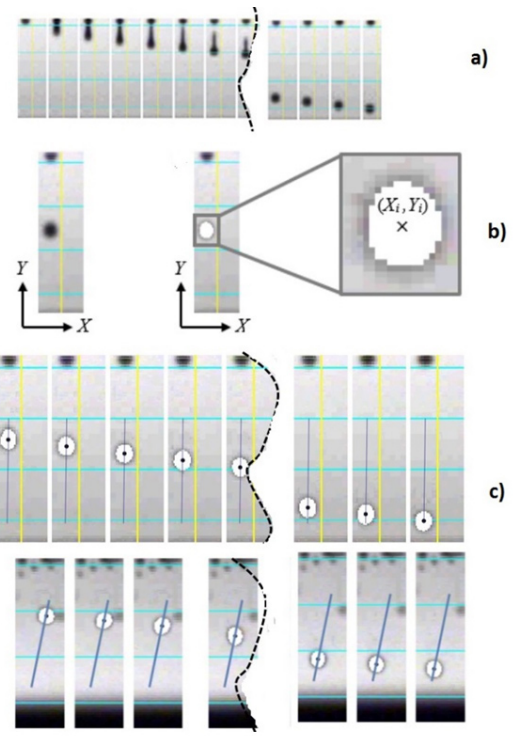

Figure 6: Droplet ejection during the inkjet printing process.a) image processing from original image to binary image with white coloured foreground pixels and schematic representation of the droplet centroid, and c) droplet centroids ejected from clean nozzle following a linear trajectory with droplet centroids from a nozzle that has been used for a few days

Following the binary conversion, morphological analysis was performed to define the droplet centroid coordinates for the $\mathrm{i}^{\text {th }}$ frame as:

$\left(X_{i}, Y_{i}\right)=\left(\frac{\sum_{j=1}^{n} x_{j}}{n}, \frac{\sum_{j=1}^{n} y_{j}}{n}\right)$

in which $\left(x_{j}, y_{j}\right)$ are the coordinates of the jth foreground pixel and $n$ is the total number of foreground pixelsinsidetheinvestigated dropletin Fig. $6 c)$. As seen in Fig. 6c) for the linear drop trajectory the fitted polynomial, i.e. $Y=74.16 X-720.11$ with angular difference $\theta_{\text {ref }}=0.77^{\circ}$ was calculated while for unlinear drop trajectory polynomial was $Y=4.91 X-29.77$ with angular difference, $\Delta \theta=\left|\theta-\theta_{\text {ref }}\right|=10.74^{\circ}$ which is giving significant difference in position where dot actually consolidates. 


\section{CONCLUSION}

Three different IP inks for functional printing on electronic devices were fabricated from different organic solvents, with different physical properties. Their dilatant rheological behaviour was found to have a deleterious effect on printing performance, and further cause clogging of the inkjet nozzles, which resulted in a change of ejected ink droplet trajectory. For the first time, deviation angle of ink trajectory has been measured using image processing. By carful tailoring of solvent used for preparation of ink deleterious dilatant behaviour of inks can be reduced, and so provides potential for further development of DoD-IP processes for functional printing.

\section{REFERENCES}

1) Bansal N. P, Singh J. P., Schneider H, Innovative processing and synthesis of ceramics, glasses and composites (VIII), Proceedings of 106th Annual Meeting of the American Ceramic Society, Indiana, USA (2004).

2) Barnes H. A. (1989) Shear thickening ("dilatancy") in suspensions of non-aggregating solid particles dispersed in Newtonian liquids, Journal of Rheology (33) 329-366

3) Cesarano III, J., Segalman, R., Calvert, P. (1998). Robocasting provides moldless fabrication from slurry deposition. Ceramic Industry, 148(4) 94-102.

4) Conrad, J. C., Ferreira, S. R., Yoshikawa, J., Shepherd, R. F., Ahn, B. Y., Lewis, J. A. (2011). Designing colloidal suspensions for directed materials assembly. Current Opinion in Colloid \& Interface Science, 16(1), 71-79.

5) Derby, B. (2010). Inkjet printing of functional and structural materials: fluid property requirements, feature stability, and resolution. Annual Review of Materials Research, 40, 395-414.

6) Derby B., Reis N. (2003). Inkjet printing of highly loaded particulate suspensions. MRS bulletin, 28(11), 815-818

7) Gardini H., Matteucci F, .Blosi M, Costa A. L, Dondi M., Galassi C. , .Raimondo M., .Baldi G., Cinottti E. (2006) Chemico-physical properties of nano-sized ceramic inks for ink-jet printing, In Qualicer 2006, IX World Congress on Ceramic Tile Quality (Vol. 3).

8) Hoath, S. D., Vadillo, D. C., Harlen, O. G., McIlroy, C., Morrison, N. F., Hsiao, W. K., Jung S, Martin G.D., Hutchings, I. M. (2014). Inkjet printing of weakly elastic polymer solutions. Journal of Non-Newtonian Fluid Mechanics, 205, 1-10.
9) Hoth, C. N., Choulis, S. A., Schilinsky, P., Brabec, C. J. (2007) High photovoltaic performance of inkjet printed polymer: fullerene blends. Advanced Materials, 19(22), 3973-3978.

10) Jang, D., Kim, D., Moon, J., Influence of Fluid Physical Properties on Ink-Jet Printability, Langmuir, 2009, 25 (5), pp 2629-2635

11) Karakoç, A, Freund, J. (2013). A direct simulation method for the effective in-plane stiffness of cellular materials. International Journal of Applied Mechanics 5 (3): 1350034 (13 pages).

12) Lewis, J. A., Smay, J. E., Stuecker, J., Cesarano, J. (2006). Direct Ink Writing of Three Dimensional Ceramic Structures. Journal of the American Ceramic Society, 89(12), 3599-3609.

13) Le, H. P. (1998), Progress and trends in inkjet printing technology, Journal of Imaging Science and Technology, 42(1), 49-62.

14) Morrison, N. F., Harlen, O. G. (2010). Viscoelasticity in inkjet printing. Rheologica acta, 49(6), 619-632.

15) Nahid, M. M., (2012), In Quest of Printed Electrodes for Light-emitting Electrochemical Cells: A comparative Study between Two Silver Inks, Master Thesis 12, 10 Umeå University

16) Sezgin, M., Sankur, B., (2004) "Survey over image thresholding techniques and quantitative performance evaluation", 13 (1) 146-168

17) Shore, H. J., Harrison, G. M., (2005). The effect of added polymers on the formation of drops ejected from a nozzle. Journal of Electronic Imaging 13(1): 146-165.

18) Reitz, T. L., Miller, R. L., (2009) Ink-jet Printing: a versatile method for multilayer solid oxide fuel cells fabrication (Postprint), Journal of the American Ceramic Society 92. 12: 2913-2919.

19) Tellier, J., Malic, B., Kuscer, D., Trefalt, G., Kosec, M., Ink-Jet Printing of In2O3/ZnO

20) Teng, W. D, Edirisinghe, M. J., Evans, J. R. G., (1997) Optimization of Dispersion and Viscosity of a Ceramic Jet Printing Ink, Journal of American Ceramic Society, 80(2), 486-494.

21) Voigt, M. M., Mackenzie, R. C. I., King, S. P., Yau, C. P., Atienzar, P., Dane, J., Keivanidis, P. E., Zadrazil, I., Bradley, D. D. C., Nelson, J., (2012) Gravure printing inverted organic solar cell: the influence of ink properties on film quality and device performance, Solar Energy Materials and Solar Cells, 2012; 105: 77-85.

Paper sent to revision: 21.09.2015.

Paper ready for publication: 16.12.2015. 